\title{
Semillas, hijos y pueblos: cuando la maternidad se conforma en lucha
}

Seeds, children and peoples: when motherhood is formed in struggle

\section{Silvana Sciortino}

\section{(2) OpenEdition \\ 1 Journals}

\section{Electronic version}

URL: http://journals.openedition.org/corpusarchivos/1857

DOI: 10.4000/corpusarchivos. 1857

ISSN: 1853-8037

\section{Publisher}

Diego Escolar

\section{Electronic reference}

Silvana Sciortino, «Semillas, hijos y pueblos: cuando la maternidad se conforma en lucha », Corpus [En línea], Vol. 7, No 1 | 2017, Publicado el 30 junio 2017, consultado el 10 diciembre 2020. URL http://journals.openedition.org/corpusarchivos/1857; DOI : https://doi.org/10.4000/corpusarchivos. 1857

This text was automatically generated on 10 December 2020. 


\title{
Semillas, hijos y pueblos: cuando la maternidad se conforma en lucha ${ }^{1}$
}

\author{
Seeds, children and peoples: when motherhood is formed in struggle
}

\author{
Silvana Sciortino
}

1 La temática que convoca este debate me invita en lo personal a volver sobre aspectos trabajados en mi investigación doctoral sobre políticas de identidad y prácticas colectivas de mujeres indígenas (Sciortino 2013). En el caso estudiado, las mujeres originarias que describo se hallan participando de escenarios políticos públicos, organizadas, debatiendo entre ellas y con mujeres no indígenas. En mis estudios de posgrado retomé las voces de una multiplicidad de mujeres indígenas que, desde distintos lugares del país, comunidades de pertenencia y adscripción política afirman una identidad indígena desde su condición de mujeres. El interés investigativo no fue contar a otros públicos qué dicen las mujeres indígenas en el marco del movimiento de mujeres, sino dar cuenta de la complejidad de los procesos de organización social y política revisando enfoques hegemónicos sobre la lucha de las mujeres desde la diversidad de miradas y prácticas que el trabajo de campo puso ante mí. En este sentido, encontré, como antecedentes, líneas de pensamiento que apuestan a desarmar las tramas coloniales que perduran aún al interior de un movimiento crítico como es el feminismo y el de mujeres (Davis 2005; Sierra 1997; Suárez Navaz y Hernández 2008; Segato 2011).

2 En esta oportunidad, me detendré en una de las modalidades de afirmación identitaria desde la cual pude observar una serie de negociaciones, tensiones y acuerdos constitutivos de un "hacer juntas" (Fernández Álvarez 2016) como "mujeres de los pueblos originarios". ${ }^{2}$ Desde el caso que a continuación abordo, daré cuenta de la relevancia de marcos de pensamientos no prescriptivos sobre el hacer de las mujeres. En especial, cuando las mismas nos encontramos en el camino de revisión del lugar ocupado en el marco de nuestras comunidades, pueblos, espacios de movilización social. De este modo, los feminismos no hegemónicos nos comprometen en una práctica descolonizadora que cuestiona modelos preestablecidos de mujer, de formas de lucha y de prácticas de liberación desde experiencias y saberes situados. En este camino leeré las maternidades 
políticas que se ponen en juego en la conformación de la mujer indígena como actor político en la lucha de sus pueblos.

3 La representación de la mujer indígena en sus intervenciones públicas, así como en lo relatado en entrevistas, reúne una serie de sentidos que asocian a la mujer con su rol materno. Mi hipótesis de trabajo sostiene que esta configuración identitaria funciona de mínima en dos sentidos que requieren de lecturas políticas específicas. Por un lado, una tendencia conservadora que encuentra apoyo en el movimiento indígena global. El cual, ante el avance de las mujeres y en especial de las indígenas en la lucha por derechos específicos, habilitó una figura global de la mujer originaria asociada a su rol reproductivo (en términos biológicos y culturales). Una mujer indígena "hiperreal", "enlatada" (Ramos 1994; Segato 2007), que responde a una agenda global despolitizada y alejada de las luchas locales situadas en los países no hegemónicos. ${ }^{3}$

4 Por otro lado, y sobre lo que me ocuparé en este trabajo, pude observar una afirmación de la mujer indígena como madre que se define en términos de una maternidad colectiva. Este sentido trasgrede las dicotomías varón-político/mujer-doméstica que muchas de sus hermanas vienen denunciando al interior del movimiento indígena cuando no se les permite ocupar lugares de liderazgo o toma de la palabra, entre otras (Sciortino 2014).

En la construcción de esta forma de identificación se tensan y negocian determinados sentidos asociados a la mujer, al varón, a sus roles y ámbitos de trabajo. Una primera noción refiere a la complementariedad ${ }^{4}$ a la cual refieren para describir la forma de relación entre los géneros inherente a sus pueblos. En especial, con la misma sostienen una simetría en las relaciones de género respecto de las tareas cotidianas del trabajo y de los roles en el hogar. El vínculo complementario con el varón compromete a la mujer a determinadas actividades y funciones en el grupo comunitario y familiar. La complementariedad, como me fue explicado, implica "equilibrio" y "armonía" entre varones y mujeres. La mujer originaria, en este vínculo complementario con el varón, es la encargada de la reproducción. La naturaleza resulta el fundamento del rol femenino:

La mujer indígena es como la Pachamama, como la tierra, es la que da, la que procrea, la que cuida. Cuando se levantan las cosechas, la mujer es la que tiene que guardar la semilla. De esas buenas semillas, las que se guardaron para la nueva siembra es de donde va a haber de nuevo los buenos frutos. [...]Porque es como esa semilla [se refiere al hijo], la mujer tiene que saber dejar a sus hijos porque esos hijos son los que van a seguir. ${ }^{5}$ (El resaltado me pertenece)

Directamente unida a la complementariedad entre los sexos se reivindica la figura de la mujer-madre, la cual se fundamenta en la analogía entre mujer y naturaleza. En sus discursos, las mujeres se identifican con la naturaleza, "la Pachamama", "la Ñukemapu", son mujeres que "dan vida". A partir de poseer esta capacidad (similar a la naturaleza, a la tierra) se asocian al cuidado de los hijos/as ("las semillas"). En los relatos recabados la naturaleza fundamenta los roles y obligaciones de cada género. Más allá de su pertenencia étnica específica, las mujeres indígenas en sus intervenciones (aunque en algunos relatos se extiende a la mujer en términos universales) se afirman nutricias, dadoras de vida, cuidadoras del grupo familiar.

7 Partiendo de esta analogía mujer-madre, uno de los sentidos que se reivindica es el de una mujer-madre como "trasmisora de cultura", "cuidadora de los hijos", destacando que estas funciones las ubican en el espacio doméstico: "la mujer es el núcleo fundamental, el hogar". Como me dijo una de las mujeres entrevistadas: 
Alcanzar el equilibrio ${ }^{6}$ es preguntarme ¿Qué es lo que yo quiero de mí? ¿Cómo quiero vivir como mujer? ¿Cómo voy a formar el hogar? Porque la mujer es la que va a armar el hogar. [...] La mujer cumple una función muy importante y este sistema donde el feminismo dispersa sus energías por cualquier lado y no cumplen esa función. Están pensando en algo que no es bueno. Como Pueblos originarios somos más madres, no abandonamos a nuestros hijos. ${ }^{7}$ (El resaltado me pertenece)

En esta perspectiva se expone un ideal de mujer-madre basado en la representación de una madre abnegada, presente y principal responsable del cuidado de los/as hijos/as. Desde este posicionamiento sobre la maternidad se conforma un estereotipo que reduce la pluralidad de vínculos que la maternidad refleja en diversas sociedades indígenas. Esta tendencia que describí como conservadora, también se presenta clausurando debates sobre cuestiones que hacen a la salud reproductiva y a situaciones de violencia contra las mujeres (Sciortino 2014).

Dada las posibilidades de extensión, quiero ocuparme aquí de la reivindicación de la mujer indígena como madre en un sentido que considero desafiante respecto a los estereotipos de género antes mencionados. De manera reiterada en el registro, la mujer originaria aparece asociada a la naturaleza, la maternidad, la crianza. Pero también a la "fuerza", a la trasmisión de la vida y de la cultura. La mujer es la que contiene, como hace la naturaleza con todos los seres, a los hijos de su pueblo al ser las encargadas de trasmitir "la cultura":

El hombre que nosotras tenemos que formar es el hombre que nos apoye, la mujer es la que tiene que luchar por la tierra, la mujer tiene que arraigar al hijo a su tierra, la mujer tiene que enseñar que nosotros somos capaces, que siempre hemos vivido aún en el destierro de mi pueblo, aún en la matanza de hoy, aún en las mentiras de hoy somos capaces de vivir dignamente si nos dan la tierra. ${ }^{8}$ (El resaltado me pertenece)

Podemos observar una tendencia diferente que pone en juego categorías como la de "lucha" en la conformación de las representaciones de la mujer indígena en discursos reivindicatorios. Una mujer activa que forma y enseña en pos de la lucha por la tierra y la vida digna. Aquí, aunque aun enunciando la figura de la mujer-madre, no se manifiesta contraposición al rol materno ante la participación de las mujeres en la lucha política. En sus intervenciones la mujer-madre se enarbola al mismo tiempo como mujer "luchadora", "guerrera":

El Pueblo mapuche no tenía ejército mixto, tenía ejército de mujeres y ejército de hombres. Y las mujeres desarrollaban sus estrategias militares de acuerdo a su condición física, de acuerdo a los beneficios que la naturaleza le había dado. [...] Entonces a veces decimos, nos complementamos con el hombre porque el hombre tiene fuerza, porque el hombre sabe hacer cosas que nosotras no podemos. Nosotras podemos enfrentar la misma problemática que un hombre pero con otras capacidades y otro desarrollo. ${ }^{9}$

11 En palabras de Moira, la maternidad no la distancia de la lucha:

les pido ayuda por otra lucha, por otra bandera que quiero levantar y es la plenitud de la maternidad de quienes elegimos ser madres. Porque mucha sociedad capitalista, depredadora, contaminante, este sistema genocida nos permite el derecho a ser madres (según ellos) pero no podemos nosotras ejercer la plenitud de nuestra maternidad porque nos pasamos veinte horas trabajando para el capitalismo y muchas veces no podemos ver crecer a nuestros hijos. Y también es una lucha poder vivir con plenitud nuestra maternidad (El resaltado me pertenece). ${ }^{10}$

12 En concordancia con estos relatos, recabé en mi experiencia de campo referencias a mujeres indígenas reivindicadas como "luchadoras". A los nombres de mujeres como 
Bartolina Sissa y Micaela Bastidas, Gerónima Sande, Ayme Payne, Ramona Quiroga, Lucinda Quintipurray, Luisa Calcumil se suman las de Esperanza Nieva y Viviana Coliqueo.

La mujer-madre es la "fuerza" que garantiza la perduración de las comunidades, en términos biológicos y culturales, resguardado el conocimiento y trasmitiéndolo a sus hijos/as. Desde una representación asociada a la tierra, la mujer originaria se afirma "luchadora" y, como fue posible observar, también como interlocutora en espacios de debate político frente a movimientos sociales, instituciones del Estado, organismos internacionales. La maternidad y sus responsabilidades asociadas (en tanto que trasmisoras de la cultura, el conocimiento, la vida, la educación, las costumbres) proveen un modelo para entrar en la lucha política pública de sus pueblos. Como lo hace en el núcleo del hogar cuidando a sus hijos y grupo familiar, la mujer construye protagonismo tomando la palabra y debatiendo en los espacios de movilización social. Ellas trasforman la participación invisibilizada que algunas vienen denunciando a partir de una correlación de responsabilidades entre lo doméstico y lo público.

da mujer indígena y la forma en que se presentan como interlocutoras en los espacios políticos se fundamenta en un entramado de sentidos que asocia a la mujer, la complementariedad y la naturaleza. Entre las representaciones relevadas en torno a la figura de la mujer indígena pude observar dos posiciones tendientes a trasladar el lenguaje de la domesticidad a la política. Ambas reafirman la condición de mujer-madre. Pero mientras una la coloca en la escena política con el fin de defender un estereotipo de mujer-madre que congela las potencialidades de las mujeres a su rol de cuidadora abnegada y "núcleo del hogar", la segunda habilita a las luchas sociales y a la ocupación del espacio político público en tanto madres de un colectivo, una mujer que "lucha" y "cuida" de su pueblo.

15 A partir de lo descrito, la noción de "madre" se entiende como constructo político que adquiere sentido en una trama amplia donde se establecen roles de género y responsabilidades en la sociedad y en la familia. También en la organización colectiva. Si los roles de la mujer como madre, cuidadora y trasmisora se trasladan a un lenguaje político que define a una madre colectiva, de hijos que "son los que van a seguir", que son el pueblo, hallan una modalidad de participación política pública que no las enfrenta a los lineamientos conservadores de sus propios movimientos. Conservan su lugar nutricio, pero ahora en el marco de la organización y movilización social. Ellas se reivindican frente a otros sectores sociales, en especial frente a otras mujeres, como las indicadas para resguardar con su lucha el "conocimiento", los "saberes" y la "cultura" de sus comunidades porque así lo hacen en sus familias.

En una de sus intervenciones en el taller de "Mujeres de los Pueblos originarios" en el Encuentro Nacional de Mujeres realizado en la ciudad de Neuquén (2008), una mujer mapuche interpeló a sus hermanas presentes: “¿Cuál será el lugar de las mujeres en los procesos de liberación?". Estas madres luchadoras pueden ser un momento de un tenso y complejo proceso que implica desarmar tramas opresivas y cuestionar lugares, roles, capacidades, muchas de las veces naturalizadas en términos de "costumbres ancestrales" (Falquet 2001; Sierra 1997). La organización de las indígenas a partir de su afirmación como mujeres originarias conlleva la vivencia de una serie de tensiones y conflictos intra e intergrupales. Ellas relatan el resquemor de los líderes varones al momento en que deciden organizarse y tomar la palabra. También refiere a los conflictos que se generan con sus hermanas indígenas al cuestionar roles y relaciones entre los géneros. 
17 Cabe destacar también las tensiones generadas cuando la organización colectiva se construye en espacios de intercambio con otros sectores de mujeres. Las feministas conformamos un espacio con el cual las originarias vienen dialogando directa e indirectamente. Los momentos de desacuerdo o de dificultad para mantener el diálogo son frecuentes. En estos conflictos suelen imperar ciertos estereotipos que generan lugares de enunciación desiguales. Me refiere a una definición del feminismo como "occidental", "de mujeres blancas", "colonial", "racista". Frente a una mirada asistencialista, "salvacionista", que contempla a las indígenas como "pobres", "carentes de recursos", "necesitadas de ayuda".

Entre ambas posiciones emergen incipientes intervenciones y prácticas colectivas que buscan un acercamiento. Mujeres indígenas que exponen la urgencia de debatir sobre violencia, legalización del aborto, relaciones de desigualdad en el movimiento indígena, apoyándose en las luchas feministas. Pero también, feministas que nos oponemos a las tramas coloniales al interior del propio movimiento, que discutimos con las miradas monolíticas construidas sobre las mujeres, que desafiamos formulaciones moralistas, salvacionistas de quienes suelen ser definidas como "las otras".

19 Así como una parte del feminismo se replegó sobre sí mismo de manera crítica y hoy en día los feminismos no hegemónicos se encuentran en una etapa floreciente, creciendo de manera localizada (latinoamericano, comunitario, indígena, populares), considero que algunos sectores organizados de mujeres originarias se deben la tarea de desarmar ciertas "costumbres", "tradiciones" asociadas a los géneros, las mujeres, la familia. Así también correrse de una mirada sobre el feminismo muchas veces fundada en el desconocimiento o en el sentido común que suele prevalecer sobre el mismo. Si en la construcción política de las mujeres originarias el feminismo se mantiene en el lugar de "lo otro", de la colonialidad, o la desigualdad de género no es leída en la trama de sus propios pueblos, el diálogo tiende a clausurarse.

La convicción por un saber y una práctica política descolonizadora puede pensarse como un puente entre mujeres con trayectorias diversas. En lugar de vincularnos desde el "deber ser" que nos imponen nuestros pueblos, movimientos, los lazos solidarios pueden nacer desde la comprensión de la creatividad desde la cual las mujeres hemos conseguido organizarnos, resistir, modificar vínculos opresivos. La maternidad ha funcionado para algunas mujeres como un nexo entre el trabajo reproductivo y las prácticas políticas. En mi opinión, descolonizar el feminismo implica, entre otros desafíos, reconocer las experiencias de luchas locales. Estas se configuran a partir de trayectorias individuales y colectivas situadas en contextos históricos y culturales. Desde allí, dar cuenta de la creatividad en la construcción de lenguajes políticos y de prácticas colectivas que resignifican roles de género, que han encontrado en los grupos domésticos, el trabajo reproductivo, un camino hacia la apropiación de los espacios de debate y movilización social. 


\section{BIBLIOGRAPHY}

Davis, A. (2005). Mujeres, raza y clase. Madrid: Ediciones Akal.

Falquet, J. (2001). La costumbre cuestionada por sus fieles celadoras: reivindicaciones de las mujeres zapatistas. Debate Feminista, 12(24), 163-190.

Fernández Álvarez, M. I. (Comp.). (2016). Hacer juntos(as). Dinámicas, contornos y relieves de la política colectiva. Buenos Aires: Editorial Biblos.

Martin, J. (1990). Motherhood and Power: The Production of a Women's Culture of Politics in a Mexican Community. American Ethnologist, 17 (3), 470-490.

Ramos, A. (1994). The Hyperreal Indian. Critique of Anthropology, 14 (2), 153-171.

Sciortino, S. (2015). Procesos de organización política de las mujeres indígenas en el movimiento amplio de mujeres en Argentina. Consideraciones sobre el feminismo desde la perspectiva indígena. Revista Universitas Humanística, 79, 65-87.

Sciortino, S. (2014). Violencias relatadas, derechos debatidos y mujeres movilizadas: el aborto en la agenda política de las mujeres indígenas en Argentina. Revista Caravelle, 102, 87-106.

Sciortino, S. (2013). Una etnografía en los Encuentros Nacionales de Mujeres: políticas de identidad desde la afirmación de las 'mujeres de los pueblos originarios'. Tesis de doctorado. Facultad de Filosofía y Letras. Universidad de Buenos Aires.

Sciortino, S. (2013a). Mujeres, madres y luchadoras: representaciones políticas de las mujeres originarias en los discursos identitarios. Actas VII Jornadas Santiago Wallace de Investigación en Antropología Social. En: http://www.jias.org.ar/conf-cientifica/comunicacionesActasEvento.php Segato, R. (2011). Género y colonialidad: en busca de las claves de lectura y de un vocabulario estratégico descolonial. En K. Bidaseca y V. Vázquez Laba (Comps.), Feminismo y Poscolonialidad. Descolonizando el feminismo desde y en América Latina, (pp.17-48). Buenos Aires: Ediciones Godot.

Segato, R. (2007). La Nación y sus Otros. Raza, etnicidad y diversidad religiosa en tiempos de Políticas de la Identidad. Buenos Aires: Prometeo.

Sierra, M. T. (1997). Esencialismo y autonomía: paradojas de las reivindicaciones indígenas. Alteridades, 7 (14), 131-143.

Suárez Navaz, L. R. y Hernández Castillo, A. (Eds.). (2008). Descolonizando el feminismo. Teorías y prácticas desde los márgenes. Madrid: Ediciones Cátedra.

\section{NOTES}

1. Este artículo retoma aspectos desarrollados en Sciortino 2013a

2. Así se autoproclaman en el marco de los Encuentro Nacionales de Mujeres (ENM), principal espacio relevado en mi investigación. De todos modos, esta identificación se registra trascendiendo los ENM, en otros eventos sociales y políticos. Para mayor detalle sobre los ENM y para conocer la diversidad que contiene esta categoría adsciptiva, ver Sciortino 2015.

3. Me explayo sobre esto en Sciortino 2013; 2014. 
4. Las categorías nativas se destacan en letra cursiva.

5. Mujer aymara de Bolivia. Palabras pertenecientes a la exposición que realizó en la mesa titulada "La mujer indígena hoy", en las Jornada por el mes de la memoria, FTS, UNLP, La Plata, octubre 2009.

6. Venía refiriéndose a la complementariedad como equilibrio.

7. Mujer quechua aymara de la comunidad de Qhapaj Ñan. Entrevista realizada en el marco de las Jornadas de celebración del Inti Raymi, CABA, junio 2011.

8. Esta mujer se identificó al presentarse como descendiente de uno de los últimos de los caciques de los Quilmes de la reducción de Santiago del Estero. Taller "Mujeres de los Pueblos originarios, ENM, Tucumán, 2009.

9. Mujer mapuche de la comunidad Pillán Mahuiza, Chubut. Palabras pertenecientes a la exposición que realizó en la mesa titulada "200 años de lucha y resistencia de la mujer en América Latina", "El Otro bicentenario", CABA, mayo de 2010.

10. Ver nota al pie 9.

\section{ABSTRACTS}

In this paper I reflect on a modality of political participation that indigenous women have built within the framework of social mobilization in Argentina. To this end, I dwell on the affirmation of the "original woman" as a "mother", holding that this identification operates in a minimum of two senses that require specific political readings. On the one hand, a conservative tendency that reifies indigenous women in reproductive work and domestic space. On the other hand, an affirmation of it in terms of collective motherhood which holds it as responsible for reproduction but also as a participant in the struggle of its peoples in the public political arena.

From the development of these forms of identity affirmation, I hold the relevance of nonprescriptive frames of thought about the making of women. Especially when we are in the process of reviewing the place occupied in the framework of our communities, towns, spaces of social mobilization. Non-hegemonic feminisms engage us in a decolonizing practice that challenges pre-established models of women, forms of struggle and practices of liberation from local experiences and knowledge. In this way collective maternities are understood as a creative practice built locally towards the appropriation of spaces for debate and social mobilization.

En este trabajo reflexiono sobre una modalidad de participación política que las mujeres indígenas han construido en el marco de la movilización social en Argentina. Con este objetivo, me detengo en la afirmación de la "mujer originaria" como "madre", sosteniendo que esta identificación funciona de mínima en dos sentidos que requieren de lecturas políticas específicas. Por un lado, una tendencia conservadora que reifica a la mujer indígena en el trabajo reproductivo y en el espacio doméstico. Por otro lado, una afirmación de la misma en términos de maternidad colectiva, la cual la sostiene como responsable de la reproducción, pero también como partícipe de la lucha de sus pueblos en el ámbito político público.

A partir del desarrollo de estas modalidades de afirmación identitaria sostengo la relevancia de marcos de pensamientos no prescriptivos sobre el hacer de las mujeres. En especial, cuando las mismas estamos en el camino de revisión del lugar ocupado en el marco de nuestras comunidades, pueblos, espacios de movilización social. Los feminismos no hegemónicos nos comprometen en una práctica descolonizadora que cuestiona modelos preestablecidos de mujer, 
de formas de lucha y de prácticas de liberación desde experiencias y saberes situados. En este camino las maternidades colectivas son comprendidas en tanto práctica creativa construida localmente en pos de la apropiación de los espacios de debate y movilización social.

INDEX

Palabras claves: Mujeres indígenas, feminismos no hegemónicos, maternidades colectivas, prácticas políticas

Keywords: Indigenous women, non-hegemonic feminisms, collective maternities, political practices

\section{AUTHOR}

\section{SILVANA SCIORTINO}

Consejo Nacional de Investigaciones Científicas y Técnicas, Instituto de Investigaciones en Humanidades y Cs. Sociales, Universidad Nacional de La Plata, Argentina

Correo electrónico: silvana.sciortino@gmail.com 